\title{
Regressing Cutaneous Melanoma
}

National Cancer Institute

\section{Source}

National Cancer Institute. Regressing Cutaneous Melanoma. NCI Thesaurus. Code C7089.

Cutaneous melanoma characterized by the disappearance of the melanoma cells from the primary tumor. 